\title{
RELATION BETWEEN SUBJECTIVE AND PHYSICAL WELL-BEING AND MINDFULNESS
}

\author{
Lisa A. Best, Cecile J. Proctor, Tracy A. Freeze, Derek J. Gaudet, Ryley Russell, \\ \& Rory McPhee \\ Department of Psychology, University of New Brunswick (Canada)
}

\begin{abstract}
An individual's sense of well-being involves the complex interaction of psychological and health-related quality of life. Satisfaction with Life is reported subjectively and encompasses cognitive (assessment of life circumstances) and emotional (assessment of negative emotions) factors (Tay, Kuykendall, \& Diener, 2015). Physical health is a more objective measure of overall physical and emotional functioning, social engagement, emotional well-being, energy levels, fatigue, pain, and general health perceptions (Hays \& Morales, 2001). There is increasing evidence that mindfulness is associated with psychological and physical outcomes (Ludwig \& Kabat-Zinn, 2008). Our purpose was to examine how different aspects of mindfulness (observing, describing, acting with awareness, non-judging of inner experience, and non-reactivity to inner experience) were related to physical and psychological well-being. In total, 513 non-clinical undergraduate participants completed questionnaires to measure life satisfaction, physical and psychological wellness and mindfulness. The current results highlight how personality and mindfulness affect both physical and psychological wellness. Specifically, emotional stability, extraversion, conscientiousness, and agreeableness were associated with better health outcomes and increased mindfulness. Some aspects of mindfulness (awareness and non-judging) were associated with both physical and psychological health. Given these results, we would suggest that individuals interested in improving their physical and psychological health might attend to an increased and non-judgemental focus on acting in the present moment.
\end{abstract}

Keywords: Five factor mindfulness, personality, psychological wellness, physical wellness.

\section{Introduction}

General well-being involves the complex interaction of psychological and health-related quality of life (Diener, Emmons, Larsen, \& Griffin, 1985). Physical health is an objective measure of overall physical and emotional functioning, social engagement, emotional well-being, energy levels, fatigue, pain, and general health perceptions (Hays \& Morales, 2001). In health-related research, it is common to focus on the measurement of specific mental health problems (i.e., anxiety, depression) in order to determine if psychological symptomology is co-morbid with specific medical conditions and their associated symptoms (i.e., limited mobility, pain). Satisfaction with life is reported subjectively and encompasses cognitive (assessment of life circumstances) and emotional (assessment of negative emotions) factors (Tay, Kuykendall, \& Diener, 2015). Measures of life satisfaction focus on the overall perception of one's life, with specific measures focused on the subjective experience of "happiness".

\subsection{Mindfulness}

Mindfulness is been shown to ameliorate work stress (Bostock, Crosswell, Pratha, \& Steptoe, 2018), and depression ( $\mathrm{Li} \&$ Bressington, 2019). Marzabadi, Mills, and Valikhani (2018) reported significant differences in the levels of depression, anxiety, stress, physical and psychological health among those with low, medium, and high levels mindfulness. Further, there are significant associations between symptoms of psychological disorders (Obesssive Compulsive, Major Depression, Borderline Personality Disorder) and facets of mindfulness, indicating that those suffering from psychological disorders practice lower levels of mindfulness compared with healthy controls. Overall, research suggests that the ability to be present in the moment and aware of personal emotions without reaction and judgement has impacts across physical and psychological domains of well-being.

Research on mindfulness has suggested that there are five factors that contribute to being mindful: Observing; Describing; Acting with awareness; Non-judging of inner experience; and, Non-reactivity to inner experience (Baer et al., 2008). Observing involves attending to or taking notice of internal and external bodily experiences, including sensations, emotions, sounds, and smells. Observing involves the conscious awareness of both personal emotions (positive and negative) and environmental 
sensations (i.e., noticing how the rain sounds vs. an awareness of the fact that it is raining). Describing is being able to translate internal experiences to words (e.g., being able to differentiate between anger, frustration, and jealousy). The ability to be present in the moment is the defining quality of acting with awareness. Simply stated, acting with awareness is paying attention to a task without allowing the mind to wander. The non-judgement of inner experiences involves the ability to observe inner thoughts and feelings without placing positive or negative value onto them. The lack of judgement means that one is able to allow thoughts to come and go, without assigning a valence to them. When one avoids value labels, the avoidance of negative thoughts is unnecessary. Non-reactivity to inner experience is the ability to experience positive and negative emotion without acting in accordance with them (Baer, Smith, Hopkins, Krietemeyer, \& Toney, 2006; Baer et al., 2008).

\subsection{Personality}

The most common model of personality is the Five Factor Model, in which personality is defined by Extroversion, Agreeableness, Openness to Experience, Conscientiousness and Neuroticism (or Emotional Stability). These factors are traits that can explain and predict behavior (Costa \& McCrae, 1992). Neuroticism involves increased levels of psychological distress and unpleasant feelings and emotions. Extraversion, or sociability, is associated with higher levels of friendliness, activity, and the experience of positive emotions. Openness to experience involves intellectual curiosity, flexibility in thoughts and behaviours, and a readiness to adjust in different situations. Agreeableness involves feelings of sympathy, cooperation, and trustworthiness, with lower scores indicating distrust and pessimism. Conscientiousness is associated with an increased propensity for both organization and diligence. In adults, personality is relatively stable even during major life changes or events and affects individual coping styles (Allemand, Steiger, \& Hill, 2013). Personality factors are commonly studied in relation to well-being (Hayes \& Joseph, 2003); individuals with lower neuroticism and higher extroversion generally have higher subjective well-being (Schimmack, Radhakrishnan, Oishi, Dzokoto, \& Ahadi, 2002). Additionally, individuals who suffer from depression report higher levels of emotional instability and lower mindfulness indicating possible crossover between these factors and the relation to psychological health.

\section{Purpose of the current study}

Our overall goal was to examine how mindfulness (observing, describing, acting with awareness, non-judging of inner experience, and non-reactivity to inner experience) was related to psychological and physical health. Further, we were interested in examining if personality factors (emotional stability, extraversion, conscientiousness, agreeableness, openness to experience) were related to specific aspects of mindfulness and overall wellness.

\section{Methods}

In total, 513 undergraduate student participants volunteered to complete an online questionnaire package and received course credit for participation. Participants ranged in age from 17 to 52 years $(M$ age $=20.55, S D=5.13)$. Approximately half of the participants reported being in a romantic relationship; $65 \%$ of these participants reported that their relationship had lasted more than a year. The questionnaire package included measures of personality (BFI-2; Soto \& John, 2017), psychological well-being (Satisfaction with Life Scale, SWLS; Diener et al., 1985), general health (RAND-36 it Health Survey; Hays \& Morales, 2001), common life stressors (Social Readjustment Rating Scale, SRRS; Holmes \& Rahe, 1967), and mindfulness (Five Factor Mindfulness Questionnaire; Baer et. al., 2006).

\section{Results}

The RAND-36 subscales range from 0 (worst possible health) to 100 (best possible health). Descriptive statistics are presented in Table 1. To test specific differences between our sample and published norms, one sample t-tests were conducted. Overall, current participants had higher scores on scales associated with overall physical health and lower scores on scales associated with emotional and social well-being. Further, participants in this sample reported a variable amount of stress, with some reporting stress at levels that could impact their physical health (Holmes \& Rahe, 1967). Although the stress reported was high, it is important to note that the SRRS includes items that indicate stress associated with positive (i.e., getting married) and negative (i.e., death of a family member) events. Given that the majority of participants likely faced many changes associated with entering adulthood, these high and variable scores were expected. To highlight further that not all stress leads to decreases in life satisfaction, it is important to note that the mean SWLS indicated average/above average life satisfaction, which are typical of individuals who live in economically developed nations. More than half of the current participants had SWLS scores that indicated high satisfaction, suggesting that they have an 
enjoyable life, in which their needs are being fulfilled (Diener, n.d.). Overall, these results are not surprising. The young adults who participated in this study reported excellent physical health but are experiencing some emotional and social struggles in response to the increased responsibilities associated with young adulthood.

Table 1. Average Physical and Psychological Well-Being of Participants.

\begin{tabular}{|c|c|c|c|c|c|}
\hline \multirow[b]{2}{*}{ Rand-36 Subscale } & \multicolumn{2}{|c|}{$\begin{array}{l}\text { Current Sample } \\
(\mathrm{N}=405)\end{array}$} & \multicolumn{2}{|c|}{$\begin{array}{c}\text { Published Norms } \\
(\mathrm{N}=2471)\end{array}$} & \multirow[b]{2}{*}{ t-score } \\
\hline & Mean & SD & Mean & SD & \\
\hline Physical Functioning & 89.92 & 17.77 & 70.61 & 27.42 & $21.84 * * *$ \\
\hline Energy/Fatigue & 42.65 & 19.96 & 52.15 & 22.39 & $-9.58 * * *$ \\
\hline Emotional Well-Being & 58.13 & 20.87 & 70.38 & 21.97 & $-11.10 * * *$ \\
\hline Social Functioning & 74.51 & 24.03 & 78.77 & 25.43 & $-3.57 *$ \\
\hline Pain & 79.56 & 19.11 & 70.77 & 25.46 & $9.26^{* * *}$ \\
\hline Physical Limitations & 82.22 & 29.73 & 52.97 & 40.78 & $19.80 * * *$ \\
\hline Emotional Limitations & 59.78 & 41.81 & 65.78 & 40.71 & $-2.88 *$ \\
\hline General Health & 64.55 & 21.19 & 56.99 & 21.11 & $7.18^{* *}$ \\
\hline SRRS Total Score & 235.75 & 125.95 & & & \\
\hline SRRS Total Stressors & 9.04 & 4.49 & & & \\
\hline Satisfaction with Life & 23.65 & 6.73 & & & \\
\hline
\end{tabular}

Correlational analyses indicated statistically significant positive associations between the RAND Health Scores and the mindfulness factors (see Table 2). Overall, the Describing, Awareness, and Non-judging factors were consistently associated with physical and psychological wellness. As would be expected, the magnitude of the correlations between mindfulness and psychological health were larger than those between mindfulness and physical health.

Table 2. Relation between Mindfulness Factors and Indicators of Physical and Psychological Wellness.

\begin{tabular}{|c|c|c|c|c|c|}
\hline & \multicolumn{5}{|c|}{ Mindfulness Factors } \\
\hline & Observing & Describing & Awareness & Non- Judging & Non-reactive \\
\hline Physical Limitations & -.022 & $.134 *$ & $.162 * *$ & $.158 * *$ & .060 \\
\hline Emotional Limitations & -.076 & $.183 * * *$ & $.367 * * *$ & $.318 * * *$ & $.144 *$ \\
\hline Physical Functioning & .061 & $.141 *$ & $.127 *$ & .089 & .085 \\
\hline Energy & .013 & $.278 * * *$ & $.431 * * *$ & $.383 * * *$ & $.226 * * *$ \\
\hline Emotional Well-Being & -.003 & $.340 * * *$ & $.492 * * *$ & $.456 * * *$ & $.339 * * *$ \\
\hline Social Functioning & -.033 & $.205 * * *$ & $.338 * * *$ & $.342 * * *$ & $.198 * *$ \\
\hline Pain & $.183^{* *}$ & $.264 * * *$ & $.209 * * *$ & $.126 * * *$ & $.387 * * *$ \\
\hline General Health & $.195 * *$ & $.320 * * *$ & $.211 * *$ & $.168 * *$ & $.336 * * *$ \\
\hline SRRS (\# Stressors) & $.177 * * *$ & .011 & -.099 & $-.176^{* * *}$ & .074 \\
\hline Satisfaction with Life & $.262 * *$ & $.329 * *$ & $.359 * *$ & $.276^{* *}$ & $.233 * *$ \\
\hline
\end{tabular}

There were consistent low to moderate correlations between mindfulness and personality (see Table 3). In general, having high levels of factor and facet Extraversion, Agreeableness, Conscientiousness and low factor and facet Emotional Instability were associated with higher Mindfulness Describing, Awareness, and Non-Judging. The Observing factor of Mindfulness was associated with higher Agreeableness, Conscientious, and Openness. It is interesting to note that individuals who reported higher levels of Neuroticism had lower scores on Describing, Awareness, Non-Judging and Non-Reacting Mindfulness. This suggests that the inability to regulate one's emotions is associated with the inability to consider thoughtfully changing environmental circumstances.

\subsection{Does increased mindfulness predict physical and psychological health}

Two hierarchical regressions allowed us to examine if specific aspects of mindfulness predicts better physical and psychological well-being. In both regressions, age, relationship status, and unscaled SRRS were entered in Block 1 and the mindfulness factors were entered in Block 2. The first regression predicting RAND General Health was statistically significant, $\mathrm{F}(8,234)=5.10, p<.0001$, with $15.3 \%$ of the variability in physical health accounted for by the predictor variables. Block 1 demographic variables were not statistically significant. Block 2 was statistically significant $\left(\mathrm{R}^{2}\right.$ change $\left.=.13\right)$, with Mindfulness-Observing $(t=-1.71 ; \beta=-.11)$, Mindfulness-Awareness $(t=3.73 ; \beta=.27)$, and Mindfulness-Non Reacting $(t=2.09 ; \beta=.14)$ predicting RAND General Health. Thus, higher levels of 
Awareness and Non-Reacting coupled with lower levels of Observing was associated with better physical health. In the second regression, SWLS was the criterion variable, $\mathrm{F}(8,240)=10.33, p<.0001$, $\mathrm{R}^{2}=26.3 \%$. Block 1 was statistically significant and accounted for $6 \%$ of the variability in SWLS. Specifically, Relationship status $(t=-2.57 ; \beta=-.16)$ and Number of Stressors $(t=-2.72 ; \beta=-.17)$ predicted SWLS. Block 2 was also statistically significant $\left(\mathrm{R}^{2}\right.$ change $\left.=.20\right)$, with Mindfulness-Awareness $(t=2.36 ; \beta=.16)$, and Mindfulness-Non Judging $(t=3.68 ; \beta=.24)$, and Mindfulness-Non Reacting $(t=2.78 ; \beta=.17)$ contributing significantly to the model. Thus, being in a romantic relationship, having fewer life stressors, and higher scores on the Awareness, Non-Judging, and Non-Reacting factors of mindfulness contributed positively to life satisfaction.

Table 3. Relation between Mindfulness Factors and BFI-2 Factors and Facets.

\begin{tabular}{|c|c|c|c|c|c|}
\hline & \multicolumn{5}{|c|}{ Mindfulness Factors } \\
\hline & Observing & Describing & Awareness & Non-Judging & Non-Reacting \\
\hline Extraversion (E) & .041 & $.424 * * *$ & $.302 * * *$ & $.373 * * *$ & $.171 *$ \\
\hline E1 Sociability & .008 & $.271 * * *$ & $.188 * *$ & $.267 * * *$ & .094 \\
\hline E2 Assertiveness & .029 & $.414 * * *$ & $.211 * * *$ & $.290 * * *$ & $.149 * *$ \\
\hline E3 Energy Level & .068 & $.353 * * *$ & $.347 * * *$ & $.359 * * *$ & $.178 * *$ \\
\hline Agreeableness (A) & $.171 *$ & $.270 * * * *$ & $.250 * * *$ & $.245 * * *$ & $.158 *$ \\
\hline A1 compassion & .088 & $.218 * * *$ & $.113^{*}$ & $.164 * *$ & .028 \\
\hline A2 Respectfulness & $.173 * *$ & $.175 * *$ & $.279 * * *$ & $.176^{* *}$ & $.161 *$ \\
\hline A3 Trust & $.151 * *$ & $.255^{* * * *}$ & $.214 * * *$ & $.249 * * *$ & $.192 * * *$ \\
\hline Conscientiousness (C) & $.165 * *$ & $.312 * * * *$ & $.485 * * *$ & $.291 * * *$ & $.198 * * *$ \\
\hline C1 Organization & $.135^{*}$ & $.133^{*}$ & $.341 * * *$ & $.188^{*}$ & $.120 *$ \\
\hline C2 Productiveness & $.163 * *$ & $.383 * * *$ & $.466 * * *$ & $.290 * *$ & $.199 * *$ \\
\hline C3 Responsibility & .099 & $.268 * * *$ & $.385 * * *$ & $.242 * * *$ & $.173 * *$ \\
\hline Emotional Instability (N) & -.017 & $-.355 * * *$ & $-.487 * * *$ & $-.435 * * *$ & $-.473 * * *$ \\
\hline N1 Anxiety & -.002 & $-.291 * * *$ & $-.373 * * *$ & $-.312 * * *$ & $-.410 * * *$ \\
\hline N2 Depression & .012 & $-.353 * * *$ & $-.497 * * *$ & $-.485 * * *$ & $-.376 * * *$ \\
\hline N3 Emotional Volatility & -.051 & $-.292 * * *$ & $-.409 * * *$ & $-.346 * * *$ & $-.457 * * *$ \\
\hline Openness (O) & $.266 * * *$ & $.357 * * *$ & .102 & .050 & .054 \\
\hline O1 Intellectual Curiosity & $.291 * * *$ & $.344 * * *$ & .068 & .038 & .042 \\
\hline O2 Aesthetic Sensitivity & $.159 * *$ & $.181 * *$ & -.027 & -.055 & -.045 \\
\hline O3 Creative Imagination & $.208 * * *$ & $.359 * * *$ & $.222 * * *$ & $.153 * *$ & $.149 * *$ \\
\hline
\end{tabular}

\section{Discussion and conclusions}

The comparisons between the current study and published norms elucidate potential stressors associated with young adulthood. As would be expected of young university students, participants in the current study were in good physical health. Scores on subscales measuring physical functioning, pain, physical limitations, and general physical health were higher than published norms. Conversely, the current participants had scores indicative of lower emotional well-being; scores on RAND scales measuring emotional well-being, social functioning, limitations on emotional functioning, and energy/fatigue were lower than published norms. In addition, participants reported that they had encountered a large number of stressors over the past year and, given that the first years of university represent a transitional period between childhood and adulthood, the increase in stressors is not surprising (Denovan \& Macaskill, 2017). In spite of the lower emotional and social functioning, participants in the current sample had above average satisfaction with life, which was indicative of Canadian undergraduate students (Fowler, Davis, Both \& Best, 2018).

Overall, our results highlight that specific aspects of mindfulness are associated with both physical and psychological health. Both correlational and regression analyses suggested that higher levels of Awareness and Non-Reacting were associated with physical and psychological wellness. The current research confirms that higher levels of mindfulness is associated with increased emotional regulation, decreased rumination, and non-attachment with psychological distress (Baer, 2007; Coffey, Hartman, \& Fredrickson, 2010). Being able to act intentionally while avoiding the impulse to assign negative value judgements to current circumstances leads to positive health outcomes. The current results also highlight how other aspects of mindfulness differentially affects physical and psychological wellness. In this study, higher levels of mindful observing were associated with lower levels of perceived health. Further, the absolute number of stressors reported was positively related to Mindful Observing and negatively associated with Mindful Non-judging. Thus, it appears that an increased focus on one's bodily sensations may actually lead to an increased focus on negative sensations and stressors. The non-judging aspect of mindfulness was predictive of psychological well-being. Taking a non-judgmental approach to one's inner experiences would involve dealing with internal thoughts and feelings in a neutral manner, which allows one to avoid excessive rumination and a detachment from emotional distress (Coffey et al., 2010). 
To conclude, increased mindfulness has been associated with positive health outcomes. The current results expand upon previous research and highlights the interplay between personality and mindfulness. These results have important clinical applications. Extraversion, Conscientiousness, and Agreeableness were associated with higher scores on the factors of mindfulness while higher Emotional Instability was related to lower levels of mindfulness. Although personality factors are considered stable, mindfulness is amenable to change. Thus, regardless of personality, practicing mindfulness may lead to positive health changes. It is also interesting to note the complex associations between mindfulness, reported stress, and physical health; in this study, Mindful-Observing was associated with greater pain and life stressors, indicating it may bring undue attention to these ailments. Future research should examine mediation models to determine how personality and mindfulness influence physical and psychological well-being.

\section{References}

Allemand, M., Steiger, A. E., \& Hill, P. L. (2013). Stability of personality traits in adulthood: Mechanisms and implications. Geropsych: The Journal of Gerontopsychology and Geriatric Psychiatry, 26(1), 5-13. doi:10.1024/1662-9647/a000080

Baer, R. A. Mindfulness, assessment, and transdiagnostic processes. Psychological Inquiry, 18, 238-242.

Baer, R. A., Smith, G. T., Lykins, E., Button, D., Krietemeyer, J., Sauer, S., \& Williams, J. M. G. (2008). Construct Validity of the Five Facet Mindfulness Questionnaire in Meditating and Nonmeditating Samples. Assessment, 15(3), 329-342. https://doi.org/10.1177/1073191107313003

Baer, R. A., Smith, G. T., Hopkins, J., Krietemeyer, J., \& Toney, L. (2006). Using self-report assessment methods to explore facets of mindfulness. Assessment, 13(1), 27-45.

Bostock, S., Crosswell, A. D., Prather, A. A., \& Steptoe, A. (2019). Mindfulness on-the-go: Effects of a mindfulness meditation app on work stress and well-being. Journal of Occupational Health Psychology, 24(1), 127-138. https://doi.org/10.1037/ocp0000118

Coffey, K. A., Hartman, M., \& Fredrickson, B. L. (2010). Deconstructing mindfulness and constructing mental health: understanding mindfulness and its mechanisms of action. Mindfulness, 1(4), 235-253.

Costa, P. T., Jr., \& McCrae, R. R. (1992). Revised NEO Personality Inventory (NEO-PI-R) and the NEO Five-Factor Inventory (NEO-FFI) Professional Manual. Odessa, FL: Psychological Assessment Resources.

Costa, P. T., Jr., \& McCrae, R. R. (2010). Revised NEO Personality Inventory (NEO-PI-R) and the NEO Five-Factor Inventory (NEO-FFI) Professional Manual. Odessa, FL: Psychological Assessment Resources.

Denovan, A., \& Macaskill, A. (2017). Stress and subjective well-being among first year UK undergraduate students. Journal of Happiness Studies, 18(2), 505-525.

Diener, E. (n.d.). Understanding Satisfaction with Life Scores. Retrieved from: http://labs.psychology.illinois.edu/ ediener/SWLS.html (2 April 2019).

Diener, E. D., Emmons, R. A., Larsen, R. J., \& Griffin, S. (1985). The satisfaction with life scale. Journal of Personality Assessment, 49(1), 71-75.

Fowler, S. A., Davis, L. L., Both, L. E., \& Best, L. A. (2018). Personality and perfectionism as predictors of life satisfaction: The unique contribution of having high standards for others. Facets, 3(1), 227-241.

Hayes, N., \& Joseph, S. (2003). Big 5 correlates of three measures of subjective well-being. Personality and Individual Differences, 34, 723-727. DOI: 10.1016/S0191-8869(02)00057-0

Hays, R. D., \& Morales, L. S. (2001). The RAND-36 measure of health-related quality of life. Annals of medicine, 33(5), 350-357.

Holmes, T. H., \& Rahe, R. H. (1967). The Social Readjustment Rating Scale. Journal of psychosomatic research, 11(2), 213-218.

Li, S. Y. H., \& Bressington, D. (2019). The effects of mindfulness-based stress reduction on depression, anxiety, and stress in older adults: A systematic review and meta-analysis. International Journal of Mental Health Nursing. https://doi.org/10.1111/inm.1256

Marzabadi, E., Mills, P. J., \& Valikhani, A. (2018). Positive personality: Relationships among mindful and grateful personality traits with quality of life and health outcomes. Current Psychology: A Journal for Diverse Perspectives on Diverse Psychological Issues. https://doi.org/10.1007/s12144-018-0080-8

RAND Health Care (n.d.). 36-item short form survey (SF-36) scoring instructions. Retrieved from https://www.rand.org/health-care/surveys_tools/mos/36-item-short-form/scoring.htm

Soto, C. J., \& John, O. P. (2017). The next Big Five Inventory (BFI-2): Developing and assessing a hierarchical model with 15 facets to enhance bandwidth, fidelity, and predictive power. Journal of Personality and Social Psychology, 113(1), 117-143. 